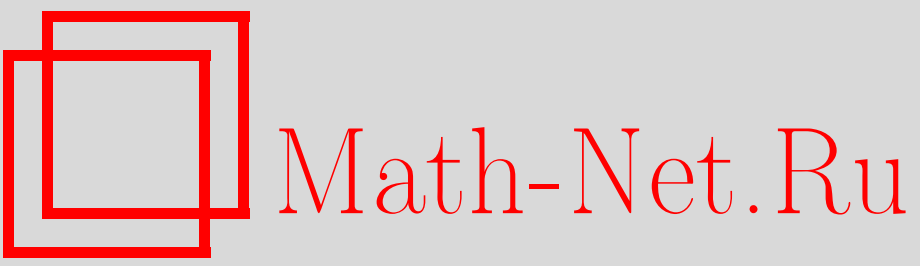

А. Г. Гаджиев, Т. Ш. Мамедов, Математические модели движущихся частиц без обгона и их приложения, Теория вероятн. и ее примен., 2011, том 56, выпуск 4, 676-689

DOI: https://doi.org/10.4213/tvp4417

Использование Общероссийского математического портала Math-Net.Ru подразумевает, что вы прочитали и согласны с пользовательским соглашением

http: //www . mathnet.ru/rus/agreement

Параметры загрузки :

IP: 54.84 .234 .179

26 апреля 2023 г., 16:40:14 
ТЕОРИЯ ВЕРОЯТНОСТЕЙ

Том 56

И ЕЕ ПРИМЕНЕНИЯ

Выпуск 4

2011

(c) 2011 г.

ГАДЖИЕВ А.Г.*, МАМЕДОВ Т.Ш.*

\title{
МАТЕМАТИЧЕСКИЕ МОДЕЛИ ДВИЖУЩИХСЯ ЧАСТИЦ БЕЗ ОБГОНА И ИХ ПРИЛОЖЕНИЯ
}

\begin{abstract}
Рассматриваются математические модели движения частиц без обгона по кольцу. Каждая частица может тормозить движение другой. Выделяются частицы-лидеры, которые могут подталкивать впереди идущие частицы, если последние тормозят их движение. Найдены необходимые и достаточные условия, при которых каждая отдельно рассматриваемая частица совершает случайное биномиальное блуждание с одинаковыми параметрами. Представлены численные результаты моделирования сложных моделей движущихся частиц, позволяющие построить диаграмму дороги в транспортных системах и выявить такие нежелательные явления, как транспортная пробка в этих системах.
\end{abstract}

Ключевые слова и фразы: движение частиц по кольцу, симметричные и несимметричные модели, лидер первого типа, лидер второго типа, абсолютный лидер, биномиальное случайное блуждание.

1. Введение. В последние годы математические модели движущихся частиц широко используются в различных приложениях. Связано это с тем, что сложные задачи, не поддающиеся исследованию аналитическими методами, могут быть изучены с помощью моделирования их поведения на компьютерах, что может дать рекомендации как для практики, так и для аналитического подхода. Прообразами моделей движения частиц по замкнутым маршрутам являются транспортные системы, биологические объекты (передача импульсов по нервным сетям), сети компьютеров, коммуникационные системы и др. Изучение математических моделей движущихся частиц позволяет выявить некоторые явления, возникающие в этих системах (возникновение «пробки» в транспортных системах [1], [2], уменьшение среднего времени ожидания требования в системах при увеличении интервалов между обслуживаниями $[3],[4$, гл.III, $\S 4$, п. г, с. 148] и др.). В [5], [6] построены математические модели движущихся частиц, для которых численно показано, что в некоторых системах увеличение интервалов между моментами начала обслуживания уменьшает среднее время ожидания требования до

\footnotetext{
* Институт кибернетики Национальной Академии наук Азербайджана, ул. Ф.Агаева, 9, Баку, AZ1141, Азербайджан; e-mail: asaf@baku-az.net, t.sh.mammadov@gmail.com
} 
обслуживания. Позже такой неожиданный результат был получен аналитическими методами для широкого класса систем [7].

В [1] построена математическая модель движения двух частиц без обгона на прямой, описывающая поведение транспортных единиц на однорядной дороге. Одна частица является лидирующей, а движение другой зависит от поведения лидера. Выявлен неожиданный эффект, когда отдельно рассматриваемая частица совершает случайное биномиальное блуждание с теми же параметрами, что и лидирующая. В [2] рассмотрена обобщенная модель движения многих частиц без обгона, где результат о случайном биномиальном блуждании используется для выявления возникновения транспортной «пробки». Близкие упрощенные модели движущихся частиц были использованы в [9] для расчета стационарных вероятностей состояний системы при движении транспортных единиц по двухполосным дорогам.

В [10], [11] исследованы различные математические модели движения частиц по кольцу и для некоторых из них также установлен факт случайного биномиального блуждания отдельно рассматриваемой частицы. Описан класс вероятностных распределений, возникающих между движущимися частицами. Показано, что произвольное дискретное распределение вероятностей можно аппроксимировать (в смысле сходимости по вариации) распределением из этого класса.

Целью настоящей работы является:

- построение математических моделей движения частиц по кольцу без обгона;

- получение необходимых и достаточных условий, при которых отдельно рассматриваемая частица совершает случайное биномиальное блуждание;

- моделирование сложных систем движущихся частиц без обгона и численный расчет некоторых характеристик (диаграмма дороги, возникновение транспортной пробки и др.).

2. Построение математической модели. Рассмотрим окружность с $n$ равноудаленными точками на ней. В некоторых выделенных точках разместим $s$ движущихся (против часовой стрелки) частиц $(s<n)$. Занумеруем точки на окружности и частицы против часовой стрелки. Движение происходит скачкообразно в моменты времени $0, h, 2 h, 3 h, \ldots(h>0)$. Каждая частица может совершить скачок вперед по направлению движения на фиксированное расстояние $d>0(d-$ расстояние между соседними точками на окружности, для простоты предполагается, что $d=1$ ) или стоять на месте.

Обозначим $\xi_{i, t}$ координаты частицы $i$ в момент времени $t$ (номер точки на окружности, которую занимает частица в момент времени $t$ ) и 
определим расстояние между частицами:

$$
\begin{aligned}
\rho_{i, t} & = \begin{cases}\xi_{i+1, t}-\xi_{i, t}, & \text { если } \xi_{i+1, t}>\xi_{i, t}, \\
n+\xi_{i+1, t}-\xi_{i, t}, & \text { если } \xi_{i+1, t}<\xi_{i, t},\end{cases} \\
\rho_{s, t} & = \begin{cases}\xi_{s, t}-\xi_{1, t}, & \text { если } \xi_{s, t}>\xi_{1, t}, \\
n+\xi_{s, t}-\xi_{1, t}, & \text { если } \xi_{s, t}<\xi_{1, t} .\end{cases}
\end{aligned}
$$

Из построения $\rho_{i, t}$ вытекают следующие свойства:

1) $1 \leqslant \rho_{i, t} \leqslant n-s+1$;

2) $\rho_{1, t}+\rho_{2, t}+\cdots+\rho_{s, t}=n$.

Введем величину $\varepsilon_{i, t}=\xi_{i, t+h}-\xi_{i, t}$, которая определяет изменение положения частицы $i$ в момент $t$ :

$$
\varepsilon_{i, t}= \begin{cases}0, & \text { если частица стоит на месте в момент } t, \\ 1, & \text { если частица совершает скачок в момент } t .\end{cases}
$$

М о д е л ь 2.1. Пусть $s=2$ и движение происходит по следующему закону:

$$
\begin{aligned}
& \mathbf{P}\left\{\varepsilon_{1, t}=1(0) \mid \rho_{1, t}=k\right\}=r_{k}\left(l_{k}\right), \\
& \quad 0<r_{k}<1, \quad r_{k}+l_{k}=1, \quad k=2, \ldots, n-1, \\
& \mathbf{P}\left\{\varepsilon_{1, t}=1(0) \mid \rho_{1, t}=1, \varepsilon_{2, t}=1\right\}=r_{1}\left(l_{1}\right), \\
& \mathbf{P}\left\{\varepsilon_{1, t}=1(0) \mid \rho_{1, t}=1, \varepsilon_{2, t}=0\right\}=0(1), \\
& \mathbf{P}\left\{\varepsilon_{2, t}=1(0) \mid \rho_{2, t}=k\right\}=r(l), \\
& \quad r>0, \quad r+l=1, \quad k=2, \ldots, n-1, \\
& \mathbf{P}\left\{\varepsilon_{2, t}=1(0) \mid \rho_{2, t}=1, \varepsilon_{1, t}=1\right\}=r(l), \\
& \mathbf{P}\left\{\varepsilon_{2, t}=1(0) \mid \rho_{2, t}=1, \varepsilon_{1, t}=0\right\}=0(1), \\
& \mathbf{P}\left\{\varepsilon_{1, t}=1(0), \varepsilon_{2, t}=1(0) \mid \rho_{1, t}=k\right\}=r_{k} r\left(l_{k} l\right), \\
& 0<r_{k}<1, \quad r_{k}+l_{k}=1, \quad k \neq 1, \\
& \mathbf{P}\left\{\varepsilon_{1, t}=1(0), \varepsilon_{2, t}=0(1) \mid \rho_{1, t}=1\right\}=l\left(l_{1} r\right) .
\end{aligned}
$$

Таким образом, если расстояние между частицами больше единицы, то сдвиги частиц происходят независимо.

Лемма 2.1. Для того чтобь для модели 2.1, описьвваемой соотношениями (2.1), вьполнялись равенства

$$
\mathbf{P}\left\{\varepsilon_{1, t}=1\right\}=\mathbf{P}\left\{\varepsilon_{2, t}=1\right\}=r
$$

необходимо и достаточно выполнение условия $r_{n-1}=1$.

Д оказатель с т в о. Д о с т а т о н о с ть. Пусть $r_{n-1}=1$. Описанная модель является эргодической цепью Маркова с конечным 
числом состояний, поэтому сущетсвует стационарное распределение [12], $\lim _{t \rightarrow \infty} \mathbf{P}\left\{\rho_{i, t}=k\right\}=a_{k}$. В [10] показано, что

$$
a_{k}=\frac{A_{k}}{A}, \quad A_{k}=\left(\frac{r}{l}\right)^{k-1} \frac{l_{1} \cdots l_{k-1}}{r_{2} \cdots r_{k}}, \quad A=\sum_{j=1}^{n-1} A_{j}, \quad A_{1}=1 .
$$

Отсюда следует рекуррентная формула

$$
a_{k} r l_{k}=a_{k+1} l r_{k+1} .
$$

Используя условие леммы $2.1\left(r_{n-1}=1\right)$ и $(2.2)$, имеем

$$
\begin{aligned}
r & =r \sum_{k=1}^{n-1}\left(r_{k}+l_{k}\right) a_{k}=\sum_{k=1}^{n-1} r r_{k} a_{k}+\sum_{k=1}^{n-1} r l_{k} a_{k} \\
& =\sum_{k=1}^{n-1} r r_{k} a_{k}+r l_{n-1} a_{n-1}+\sum_{k=1}^{n-2} r l_{k} a_{k} \\
& =r l_{n-1} a_{n-1}+\sum_{k=1}^{n-1} r r_{k} a_{k}+\sum_{k=1}^{n-2} l r_{k+1} a_{k+1} \\
& =r l_{n-1} a_{n-1}+r r_{1} a_{1}+\sum_{k=2}^{n-1} r r_{k} a_{k}+\sum_{k=2}^{n-1} l r_{k} a_{k} \\
& =r r_{1} a_{1}+\sum_{k=2}^{n-1} r_{k} a_{k} .
\end{aligned}
$$

С другой стороны,

$$
\begin{aligned}
\mathbf{P}\left\{\varepsilon_{1, t}=1\right\}= & \sum_{k=2}^{n-1} \mathbf{P}\left\{\varepsilon_{1, t}=1, \rho_{1, t}=k\right\}+\mathbf{P}\left\{\varepsilon_{1, t}=1 \rho_{1, t}=1, \varepsilon_{2, t}=1\right\} \\
= & \sum_{k=2}^{n-1} \mathbf{P}\left\{\varepsilon_{1, t}=1 \mid \rho_{1, t}=k\right\} \mathbf{P}\left\{\rho_{1, t}=k\right\} \\
& +\mathbf{P}\left\{\varepsilon_{1, t}=1 \mid \rho_{1, t}=1, \varepsilon_{2, t}=1\right\} \mathbf{P}\left\{\rho_{1, t}=1, \varepsilon_{2, t}=1\right\} \\
= & \sum_{k=2}^{n-1} r_{k} a_{k}+r_{1} r a_{1} .
\end{aligned}
$$

Следовательно,

$$
\mathbf{P}\left\{\varepsilon_{1, t}=1\right\}=r .
$$

Равенство

$$
\mathbf{P}\left\{\varepsilon_{2, t}=1\right\}=\sum_{k=2}^{n-2} r a_{k}+r r_{n-1} a_{n-1}=r\left(1-a_{n-1}\right)+r r_{n-1} a_{n-1}=r-r l_{n-1} a_{n-1}
$$


вместе с условиями $r>0, l_{n-1}=0$ и равенством (2.3) доказывают достаточность.

Н е о б х о д и м о с т ь. Пусть $\mathbf{P}\left\{\varepsilon_{2, t}=1\right\}=r$. Тогда из равенства (2.4) следует, что $l_{n-1}=0$. Аналогично доказывается случай, когда $\mathbf{P}\left\{\varepsilon_{1, t}=1\right\}=r$.

Лемма доказана.

Обозначим

$$
\varepsilon_{m}^{+}=\sum_{j=0}^{m-1} \varepsilon_{j}, \quad \varepsilon_{m}^{-}=m-\varepsilon_{m}^{+},
$$

где $\varepsilon_{i}$ принимает значения 0 или 1.

Некоторые из приводимых ниже результатов анонсированы в [13].

Теорема 2.1. Для того чтобы для модели 2.1 выполнялось равенство

$$
\mathbf{P}\left\{\varepsilon_{1, t}=\varepsilon_{0}, \varepsilon_{1, t+h}=\varepsilon_{1}, \ldots, \varepsilon_{1, t+(m-1) h}=\varepsilon_{m-1}\right\}=r^{\varepsilon_{m}^{+}} l^{\varepsilon_{m}^{-}},
$$

необходимо и достаточно выполнение условия $r_{n-1}=1$.

Д ок а з а те ль с тв о. Н е о бходи м о с т следует из леммы 2.1.

Д о с т а т о ч н о с ть. Допустим, что $r_{n-1}=1$. Покажем, что тогда для любого $m$ выполнено равенство

$$
\mathbf{P}\left\{\varepsilon_{1, t}=\varepsilon_{0}, \varepsilon_{1, t+h}=\varepsilon_{1}, \ldots, \varepsilon_{1, t+(m-1) h}=\varepsilon_{m-1}, \rho_{1, t+m h}=k\right\}=r^{\varepsilon_{m}^{+}} l^{\varepsilon_{m}^{-}} a_{k} .
$$

Воспользуемся методом математической индукции. При $m=1$ имеем

$$
\begin{aligned}
\mathbf{P}\left\{\varepsilon_{1, t}=1, \rho_{1, t+h}=k\right\}= & \mathbf{P}\left\{\varepsilon_{1, t}=1, \varepsilon_{2, t}=0, \rho_{1, t}=k+1\right\} \\
& +\mathbf{P}\left\{\varepsilon_{1, t}=1, \varepsilon_{2, t}=1, \rho_{1, t}=k\right\} \\
= & a_{k+1} l r_{k+1}+a_{k} r r_{k}=a_{k} r l_{k}+a_{k} r r_{k}=a_{k} r .
\end{aligned}
$$

Предположим, что (2.6) верно для $m$, т.е.

$$
\mathbf{P}\left\{\varepsilon_{1, t}=\varepsilon_{0}, \varepsilon_{1, t+h}=\varepsilon_{1}, \ldots, \varepsilon_{1, t+(m-1) h}=\varepsilon_{m-1}, \rho_{1, t+m h}=k\right\}=r^{\varepsilon_{m}^{+}} l^{\varepsilon_{m}^{-}} a_{k} .
$$

Докажем, что тогда (2.6) верно и для $m+1$. Имеем

$$
\begin{aligned}
& \mathbf{P}\left\{\varepsilon_{1, t}=\varepsilon_{0}, \varepsilon_{1, t+h}=\varepsilon_{1}, \ldots, \varepsilon_{1, t+(m-1) h}=\varepsilon_{m-1}, \varepsilon_{1, t+m h}=1,\right. \\
& \left.\rho_{1, t+(m+1) h}=k\right\} \\
& =\mathbf{P}\left\{\varepsilon_{1, t}=\varepsilon_{0}, \quad \varepsilon_{1, t+h}=\varepsilon_{1}, \ldots, \varepsilon_{1, t+(m-1) h}=\varepsilon_{m-1}, \rho_{1, t+m h}=k,\right. \\
& \left.\varepsilon_{1, t+m h}=1, \varepsilon_{2, t+m h}=1\right\} \\
& +\mathbf{P}\left\{\varepsilon_{1, t}=\varepsilon_{0}, \quad \varepsilon_{1, t+h}=\varepsilon_{1}, \ldots, \varepsilon_{1, t+(m-1) h}=\varepsilon_{m-1}, \rho_{1, t+m h}=k+1,\right. \\
& \left.\varepsilon_{1, t+m h}=1, \quad \varepsilon_{2, t+m h}=0\right\}
\end{aligned}
$$




$$
\begin{aligned}
&=r^{\varepsilon_{m}^{+}} l^{\varepsilon_{m}^{-}} {\left[\mathbf{P}\left\{\rho_{1, t+m h}=k, \varepsilon_{1, t+m h}=1, \varepsilon_{2, t+m h}=1\right\}\right.} \\
&\left.\quad+\mathbf{P}\left\{\rho_{1, t+m h}=k+1, \varepsilon_{1, t+m h}=1, \varepsilon_{2, t+m h}=0\right\}\right] \\
&=r^{\varepsilon_{m}^{+}} l^{\varepsilon_{m}^{-}}\left[a_{k+1} l r_{k+1}+a_{k} r r_{k}\right]=r^{\varepsilon_{m}^{+}} l^{\varepsilon_{m}^{-}} r a_{k}=r^{\varepsilon_{m}^{+}+1} l^{\varepsilon_{m}^{-}} a_{k} .
\end{aligned}
$$

Случай $\varepsilon_{1, t+m h}=0$ рассматривается аналогично. Суммируя соотношения (2.6) по $k=1, \ldots, n-1$ и учитывая, что $\bigcup_{k=1}^{n-1}\left\{\rho_{1, t+m h}=k\right\}=\Omega$ и $\sum_{k=1}^{n-1} a_{k}=1$, получаем утверждение теоремы 2.1 .

3. Общие модели движения частиц. Определим состояние системы в момент $t$ как $E_{t}=\left(\rho_{1, t}, \rho_{2, t} \ldots, \rho_{s, t}\right)$. Рассмотрим эргодические марковские модели с произвольными переходными вероятностями

$$
\mathbf{P}\left(E_{t+1}=\left(k_{1}^{\prime}, \ldots, k_{s}^{\prime}\right) \mid E_{t}=\left(k_{1}, \ldots, k_{s}\right)\right) .
$$

Теорема 3.1. Для каждой частицы в стационарном режиме выполнено равенство

$$
\mathbf{P}\left\{\varepsilon_{1, t}=1\right\}=\mathbf{P}\left\{\varepsilon_{2, t}=1\right\}=\cdots=\mathbf{P}\left\{\varepsilon_{s, t}=1\right\} .
$$

Д о к а з а т е л ь с т в о. Покажем, что для любых двух частиц $i$ и $j$ выполнено равенство $\mathbf{P}\left\{\varepsilon_{i, t}=1, \varepsilon_{j, t}=0\right\}=\mathbf{P}\left\{\varepsilon_{i, t}=0, \varepsilon_{j, t}=1\right\}$.

Для простоты положим $i=1$. Определим события

$$
\begin{gathered}
A_{m, t}=\bigcup_{k_{1}+\cdots+k_{j-1}=m}\left\{E_{t}=\left(k_{1}, \ldots, k_{s}\right)\right\}=\left\{\rho_{1, t}+\cdots+\rho_{j-1, t}=m\right\}, \\
m=j-1, \ldots, n-j+1,
\end{gathered}
$$

которые будем называть уровнями. Ясно, что $\bigcup_{m=j-1}^{n-j+1} A_{m, t}=\Omega$.

Если $k_{1}+k_{2}+\cdots+k_{j-1}=j-1$, то по формуле Колмогорова-Чепмена имеем

$$
\begin{aligned}
\mathbf{P}\left\{E_{t+h}=\left(1, \ldots, 1, k_{j}, \ldots, k_{s}\right)\right\}= & \mathbf{P}\left\{A_{j-1, t}, E_{t+h}=\left(1, \ldots, 1, k_{j}, \ldots, k_{s}\right)\right\} \\
& +\mathbf{P}\left\{A_{j, t}, E_{t+h}=\left(1, \ldots, 1, k_{j}, \ldots, k_{s}\right)\right\} .
\end{aligned}
$$

Если $k_{1}+\cdots+k_{j-1}=m(j-1<m<n-j+1)$, то

$$
\begin{aligned}
\mathbf{P}\left\{E_{t+h}=\left(k_{1}, \ldots, k_{n}\right)\right\}= & \mathbf{P}\left\{A_{m-1, t}, E_{t+h}=\left(k_{1}, \ldots, k_{n}\right)\right\} \\
& +\mathbf{P}\left\{A_{m, t}, E_{t+h}=\left(k_{1}, \ldots, k_{n}\right)\right\} \\
& +\mathbf{P}\left\{A_{m+1, t}, E_{t+h}=\left(k_{1}, \ldots, k_{n}\right)\right\} .
\end{aligned}
$$

Если $k_{1}+\cdots+k_{j-1}=n-j+1$, то

$$
\begin{aligned}
\mathbf{P}\left\{E_{t+h}=\left(k_{1}, \ldots, k_{n}\right)\right\}= & \mathbf{P}\left\{A_{n-j, t}, E_{t+h}=\left(k_{1}, \ldots, k_{n}\right)\right\} \\
& +\mathbf{P}\left\{A_{n-j+1, t}, E_{t+h}=\left(k_{1}, \ldots, k_{n}\right)\right\} .
\end{aligned}
$$


Так как $k_{1}+\cdots+k_{j-1}=m$, то

$$
\begin{aligned}
\mathbf{P}\left\{A_{j-1, t+h}\right\}= & \mathbf{P}\left\{A_{j-1, t}, A_{j-1, t+h}\right\}+\mathbf{P}\left\{A_{j, t}, A_{j-1, t+h}\right\}, \\
\mathbf{P}\left\{A_{m, t+h}\right\}= & \mathbf{P}\left\{A_{m-1, t}, A_{m, t+h}\right\}+\mathbf{P}\left\{A_{m, t}, A_{m, t+h}\right\} \\
& +\mathbf{P}\left\{A_{m+1, t}, A_{m, t+h}\right\}, \quad m=j, \ldots, n-j, \\
\mathbf{P}\left\{A_{n-j+1, t+h}\right\}= & \mathbf{P}\left\{A_{n-j, t}, A_{n-j+1, t+h}\right\} \\
& +\mathbf{P}\left\{A_{n-j+1, t}, A_{n-j+1, t+h}\right\} .
\end{aligned}
$$

Отсюда следует, что, находясь на каком-либо уровне, система может или оставаться на месте, или перейти на соседний уровень:

$$
\begin{aligned}
\mathbf{P}\left\{A_{j-1, t}\right\}= & \mathbf{P}\left\{A_{j-1, t}, A_{j-1, t+h}\right\}+\mathbf{P}\left\{A_{j-1, t}, A_{j, t+h}\right\}, \\
\mathbf{P}\left\{A_{m, t}\right\}= & \mathbf{P}\left\{A_{m, t}, A_{m-1, t+h}\right\}+\mathbf{P}\left\{A_{m, t}, A_{m, t+h}\right\} \\
& +\mathbf{P}\left\{A_{m, t}, A_{m+1, t+h}\right\}, \quad m=j, \ldots, n-j, \\
\mathbf{P}\left\{A_{n-j+1, t}\right\}= & \mathbf{P}\left\{A_{n-j+1, t}, A_{n-j, t+h}\right\}+\mathbf{P}\left\{A_{n-j+1, t}, A_{n-j+1, t+h}\right\} .
\end{aligned}
$$

Ясно, что в стационарном режиме выполнено равенство $\mathbf{P}\left\{A_{m, t+h}\right\}=$ $\mathbf{P}\left\{A_{m, t}\right\}$. Из (3.1) и (3.2) следует, что $\mathbf{P}\left\{A_{j, t}, A_{j-1, t+h}\right\}=\mathbf{P}\left\{A_{j-1, t}, A_{j, t+h}\right\}$. Таким образом,

$$
\mathbf{P}\left\{A_{m+1, t}, A_{m, t+h}\right\}=\mathbf{P}\left\{A_{m, t}, A_{m+1, t+h}\right\}, \quad m=j-1, \ldots, n-j .
$$

Так как $\mathbf{P}\left\{A_{m+1, t}, A_{m, t+h}\right\}=\mathbf{P}\left\{A_{m+1, t}, \quad \varepsilon_{1, t}=1, \quad \varepsilon_{j, t}=0\right\}$ и $\mathbf{P}\left\{A_{m, t}, A_{m+1, t+h}\right\}=\mathbf{P}\left\{A_{m, t}, \varepsilon_{1, t}=0, \varepsilon_{j, t}=1\right\}$, то (3.3) можно переписать в виде

$$
\begin{gathered}
\mathbf{P}\left\{A_{m+1, t}, \varepsilon_{1, t}=1, \varepsilon_{j, t}=0\right\}=\mathbf{P}\left\{A_{m, t}, \quad \varepsilon_{1, t}=0, \varepsilon_{j, t}=1\right\} \\
m=j-1, \ldots, n-j .
\end{gathered}
$$

С другой стороны,

$$
\begin{aligned}
\mathbf{P}\left\{\varepsilon_{1, t}=0, \varepsilon_{j, t}=1\right\}= & \sum_{m=j-1}^{n-j+1} \mathbf{P}\left\{A_{m, t}, \varepsilon_{1, t}=0, \varepsilon_{j, t}=1\right\} \\
= & \mathbf{P}\left\{A_{n-j+1, t}, \varepsilon_{1, t}=1, \varepsilon_{j, t}=0\right\} \\
& +\sum_{m=j-1}^{n-j} \mathbf{P}\left\{A_{m, t}, \varepsilon_{1, t}=1, \varepsilon_{j, t}=0\right\} .
\end{aligned}
$$

Суммируя (3.4) по $m=j-1, \ldots, n-j$ и учитывая, что $\mathbf{P}\left\{A_{j, t}, \varepsilon_{1, t}=\right.$ $\left.1, \varepsilon_{j, t}=0\right\}=0$ и $\mathbf{P}\left\{A_{n-j+1, t}, \varepsilon_{1, t}=0, \varepsilon_{j, t}=1\right\}=0$, получаем

$$
\mathbf{P}\left\{\varepsilon_{1, t}=1, \varepsilon_{j, t}=0\right\}=\mathbf{P}\left\{\varepsilon_{1, t}=0, \varepsilon_{j, t}=1\right\} .
$$

Отсюда следует, что

$$
\begin{aligned}
\mathbf{P}\left\{\varepsilon_{i, t}=1\right\} & =\mathbf{P}\left\{\varepsilon_{i, t}=1, \varepsilon_{j, t}=0\right\}+\mathbf{P}\left\{\varepsilon_{i, t}=1, \varepsilon_{j, t}=1\right\} \\
& =\mathbf{P}\left\{\varepsilon_{i, t}=0, \varepsilon_{j, t}=1\right\}+\mathbf{P}\left\{\varepsilon_{i, t}=1, \varepsilon_{j, t}=1\right\}=\mathbf{P}\left\{\varepsilon_{j, t}=1\right\} .
\end{aligned}
$$


Следствие 3.1. В стационарном режиме для любых $t_{1}, t_{2} u i, j$ выпполнено

$$
\mathbf{P}\left\{\varepsilon_{i, t_{1}}=1, \varepsilon_{j, t_{2}}=0\right\}=\mathbf{P}\left\{\varepsilon_{i, t_{1}}=0, \varepsilon_{j, t_{2}}=1\right\} .
$$

Это следует из соотношений

$$
\begin{aligned}
& \mathbf{P}\left\{\varepsilon_{i, t_{1}}=1\right\}=\mathbf{P}\left\{\varepsilon_{i, t_{1}}=1, \varepsilon_{j, t_{2}}=0\right\}+\mathbf{P}\left\{\varepsilon_{i, t_{1}}=1, \varepsilon_{j, t_{2}}=1\right\}, \\
& \mathbf{P}\left\{\varepsilon_{j, t_{2}}=1\right\}=\mathbf{P}\left\{\varepsilon_{i, t_{1}}=0, \varepsilon_{j, t_{2}}=1\right\}+\mathbf{P}\left\{\varepsilon_{i, t_{1}}=1, \varepsilon_{j, t_{2}}=1\right\} .
\end{aligned}
$$

Используя (3.4), можно найти распределение расстояния между частицами. Введем следующие обозначения:

$$
\begin{aligned}
p_{m} & =\mathbf{P}\left\{\varepsilon_{1, t}=1, \varepsilon_{j, t}=0 \mid A_{m, t}\right\}, & & m=j, \ldots, n+1-j, \\
q_{m} & =\mathbf{P}\left\{\varepsilon_{1, t}=0, \varepsilon_{j, t}=1 \mid A_{m, t}\right\}, & & m=j-1, \ldots, n-j .
\end{aligned}
$$

Так как рассматриваются эргодические модели, то (3.4) можно переписать в виде

$$
p_{m+1} \mathbf{P}\left\{A_{m+1, t}\right\}=p_{m} \mathbf{P}\left\{A_{m, t}\right\}, \quad m=j-1, \ldots, n-j .
$$

Отсюда следует, что

$$
\begin{aligned}
\mathbf{P}\left\{A_{m, t}\right\} & =\frac{q_{m-1} q_{m-2} \cdots q_{j} q_{j-1}}{p_{m} p_{m-1} \cdots p_{j+1} p_{j}} \mathbf{P}\left\{A_{j-1, t}\right\}, \quad m=j, \ldots, n+1-j, \\
\mathbf{P}\left\{A_{j-1, t}\right\} & =\left(1+\sum_{m=j}^{n+1-j} \frac{q_{m-1} q_{m-2} \cdots q_{j} q_{j-1}}{p_{m} p_{m-1} \cdots p_{j+1} p_{j}}\right)^{-1} .
\end{aligned}
$$

Таким образом, в стационарном режиме все частицы имеют одинаковую вероятность скачка. Возникает вопрос: имеет ли место для этой модели результат о случайном биномиальном блуждании отдельно рассматриваемой частицы, полученный в [1]-[3]?

4. Симметричная модель. Пусть закон движения задается следующим образом:

$$
\begin{aligned}
& \mathbf{P}\left\{\varepsilon_{i, t}=1(0) \mid \rho_{i, t}=k\right\}=r_{k}^{i}\left(l_{k}^{i}\right), \quad \begin{array}{l}
i=1, \ldots, s, \quad k=2, \ldots, n-s+1, \\
\\
r_{k}^{i}+l_{k}^{i}=1,
\end{array} \\
& \mathbf{P}\left\{\varepsilon_{i, t}=1(0) \mid \rho_{i, t}=1, \quad \varepsilon_{i+1, t}=1\right\}=r_{1}^{i}\left(l_{1}^{i}\right), \quad i=1, \ldots, s, \quad r_{1}^{i}+l_{1}^{i}=1, \\
& \mathbf{P}\left\{\varepsilon_{i, t}=1(0) \mid \rho_{i, t}=1, \quad \varepsilon_{i+1, t}=0\right\}=0(1), \quad i=1, \ldots, s .
\end{aligned}
$$

П р и м е р 4.1. Пусть $s=2$ и

$$
\begin{aligned}
r_{k}^{i}=r, \quad l_{k}^{i} & =l, \quad i=1,2, \quad k=1, \ldots, n-1, \\
r+l & =1, \quad a_{k}=\lim _{t \rightarrow \infty} \mathbf{P}\left\{\rho_{1, t}=k\right\} .
\end{aligned}
$$


Из соотношения $\sum_{k=1}^{n-1} a_{k}=1$ следует, что $a_{k}=1 /(n-1)$. Тогда

$$
\mathbf{P}\left\{\varepsilon_{1, t}=1\right\}=\sum_{k=1}^{n-1} \mathbf{P}\left\{\varepsilon_{1, t}=1, \rho_{1, t}=k\right\}=r^{2} a_{1}+\sum_{k=2}^{n-1} r a_{k}=\frac{r^{2}+(n-2) r}{n-1} .
$$

С другой стороны,

$$
\begin{aligned}
\mathbf{P}\left\{\varepsilon_{1, t}=1, \varepsilon_{1, t+h}=1\right\}= & \sum_{k=1}^{n-1} \mathbf{P}\left\{\varepsilon_{1, t}=1, \varepsilon_{1, t+h}=1, \rho_{1, t}=k\right\} \\
= & \sum_{k=1}^{n-1} \mathbf{P}\left\{\varepsilon_{1, t}=1, \varepsilon_{2, t}=1, \varepsilon_{1, t+h}=1, \rho_{1, t}=k\right\} \\
& +\sum_{k=1}^{n-1} \mathbf{P}\left\{\varepsilon_{1, t}=1, \varepsilon_{2, t}=0, \varepsilon_{1, t+h}=1, \rho_{1, t}=k\right\} \\
= & r^{4} a_{1}+\sum_{k=2}^{n-1} r^{3} a_{k}+r^{3} l a_{2}+\sum_{k=3}^{n-1} r^{2} l a_{k} \\
= & \frac{2 r^{3}+(n-3) r^{2}}{n-1} .
\end{aligned}
$$

Следовательно, $\mathbf{P}\left\{\varepsilon_{1, t}=1, \varepsilon_{1, t+h}=1\right\} \neq \mathbf{P}\left\{\varepsilon_{1, t}=1\right\} \mathbf{P}\left\{\varepsilon_{1, t+h}=1\right\}$, т.е. результат о случайном биномиальном блуждании отдельно рассматриваемой частицы в общем случае не имеет смысла.

5. Несимметричная модель. Пусть заданы $i(1 \leqslant i \leqslant s)$ и $m$ $(1 \leqslant m \leqslant s-1)$.

Обозначим $\mu_{t}(i, j)$ расстояние от $i$-й частицы до $j$-й в момент времени $t$ по направлению движения:

$$
\mu_{t}(i, j)= \begin{cases}\xi_{j, t}-\xi_{i, t}, & \text { если } \xi_{j, t}-\xi_{i, t}>0 \\ n+\xi_{j, t}-\xi_{i, t}, & \text { если } \xi_{j, t}-\xi_{i, t}<0\end{cases}
$$

Ясно, что $\mu_{t}(i, j)$ обладает следующими свойствами: 1$) 1 \leqslant \mu_{t}(i, j) \leqslant n$; 2) $\mu_{t}(i, j)+\mu(j, i)=n$; 3) $\mu_{t}(i, i+1)=\rho_{i, t}$; 4) если $i<j$, то $\mu_{t}(i, j)=$ $\rho_{i, t}+\rho_{i+1, t}+\cdots+\rho_{j-1, t}$.

Обозначим $f_{t}(i, j)$ число движущихся частиц между $i$-й и $j$-й частицами (включая $i$-ю частицу) в момент времени $t$ по направлению движения:

$$
f_{t}(i, j)= \begin{cases}j-i, & \text { если } j>i, \\ s+j-i, & \text { если } j<i .\end{cases}
$$

О п р е д е л е н и е 5.1. Будем говорить, что $i$-я частица имеет степень свободы $m$, если для любого $k \leqslant m$ выполняются следующие условия: 

$1(0)$

a) $\mathbf{P}\left\{\varepsilon_{i+k, t}=1(0) \mid \varepsilon_{i, t}=1, \mu_{t}(i, i+k)=k, \mu_{t}(i, i+m+1) \neq m+1\right\}=$

б) $\mathbf{P}\left\{\varepsilon_{i+k, t}=1(0) \mid \varepsilon_{i, t}=1, \mu_{t}(i, i+k)=k, \mu_{t}(i, i+m+1)=m+\right.$ $\left.1, \varepsilon_{i+m+1, t}=1\right\}=1(0)$;

в) $\mathbf{P}\left\{\varepsilon_{i, t}=1 \mid \varepsilon_{i+m+1, t}=0, \mu_{t}(i, i+m+1)=m+1\right\}=0$.

Другими словами, $i$-я частица имеет преимущество при движении по отношению к частицам с номерами $i+1, i+2, \ldots, i+m$, так как она подталкивает любую идущую перед ней группу частиц размера не более $m$.

О п р е д е л е н и е 5.2. Будем говорить, что $i$-я частица находится в несвободном состоянии, если выполнено хотя бы одно из следующих условий:

1) при наступлении события $A=\left\{\varepsilon_{j, t}=0, \mu_{t}(i, j)=f(i, j)\right\}$ выполняется $\mathbf{P}\left\{\varepsilon_{i, t}=1(0) \mid A\right\}=0$ (1);

2) при наступлении события $B=\left\{\varepsilon_{j, t}=1, \mu_{t}(j, i)=f(j, i)\right\}$ выполняется $\mathbf{P}\left\{\varepsilon_{i, t}=1(0) \mid B\right\}=1(0)$.

$\mathrm{B}$ противном случае частица находится в свободном состоянии.

3 а м е ч а н и е 5.1. В несвободном состоянии $i$-я частица или скачет или стоит на месте с вероятностью единица при воздействии на нее другой частицы.

О п р е д е л е н и е 5.3 . Модель движения $M\left(m_{1}, \ldots, m_{s}\right)$ назовем несимметричной, если выполняются условия:

а) первая частица имеет степень свободы $m_{1}$, вторая частица имеет степень свободы $m_{2}$ и т.д.;

б) каждая частица в свободном состоянии (когда эта частица не подталкивается и не тормозится другой) движется так же, как и в симметричной модели (вероятности скачков зависят от расстояния до следующей частицы).

Симметричная модель является частным случаем несимметричной, когда $M(0, \ldots, 0)$.

О п р е д е л е н и е 5.4. Если в модели движения $M\left(m_{1}, \ldots, m_{s}\right)$ для $i$-й частицы $(1 \leqslant i \leqslant s)$ выполняются соотношения

$$
m_{i-1}=0, \quad m_{i-2} \leqslant 1, \quad \ldots, \quad m_{i+2} \leqslant s-3, \quad m_{i+1} \leqslant s-2,
$$

то $i$-ю частицу назовем лидером первого типа, т.е. лидера первого типа не может подталкивать никакая другая частица.

О п р е д е л е н и е 5.5 . Если для модели движения $M\left(m_{1}, \ldots, m_{s}\right)$ выполнено $m_{i}=s-1$, то $i$-ю частицу назовем лидером второго типа, т.е. лидер второго типа может подталкивать все частицы.

О п р е д е л е н и е 5.6. Если для модели движения $M\left(m_{1}, \ldots, m_{s}\right)$ $i$-я частица одновременно является лидером первого и второго типов, то $i$-ю частицу назовем абсолютным лидером, и такую модель будем называть моделью движения с абсолютным лидером. 
Лемма 5.1. Пусть для модели движения $M\left(m_{1}, \ldots, m_{s}\right)$ выполненьи следуюшие условия:

1) при наступлении события $A$, для которого $i$-я частища находится в свободном состоянии, выполнено

$$
\mathbf{P}\left\{\varepsilon_{i, t}=1(0) \mid A\right\}=r(l) ;
$$

2) $i$-я частица является лидером первого или второго типа.

Тогда для того, итобь выполнялись равенства $\mathbf{P}\left\{\varepsilon_{j, t}=1(0)\right\}=$ $r(l), j=1,2, \ldots, s$, необходимо и достаточно, чтобь $i$-я частича была абсолютницм лидером.

Д о к а з а т е л ь с т в о. Д о с т а т о ч н о с т ь следует из теоремы 3.1, так как из (5.1) следует, что $\mathbf{P}\left\{\varepsilon_{i, t}=1(0)\right\}=r(l)$. Согласно теореме 3.1, для всех частиц справедливо $\mathbf{P}\left\{\varepsilon_{j, t}=1(0)\right\}=r(l)$.

Н е о б х о д и м о с т ь. Пусть $i$-я частица является лидером первого типа. Допустим, что при наступлении события $A i$-я частица находится в свободном состоянии. Тогда по условию леммы 5.1 выполнено

$$
\mathbf{P}\left\{\varepsilon_{i, t}=1(0) \mid A\right\}-r(l), \quad j=1, \ldots, s .
$$

Если при наступлении события $B i$-я частица находится в несвободном состоянии, то по построению модели имеем

$$
\mathbf{P}\left\{\varepsilon_{i, t}=1(0) \mid B\right\}=0(1) .
$$

Разлагая $\Omega$ на сумму всевозможных событий, для которых $i$-я частица находится в свободном и несвободном состояниях, и пользуясь формулой полной вероятности, получаем, что равенство $\mathbf{P}\left\{\varepsilon_{i, t}=1\right\}=r$ выполнено тогда и только тогда, когда вероятности всех событий, для которых $i$-я частица находится в несвободном состоянии, равны нулю.

Теорема 5.1. В условиях леммы 5.1 для того, чтобы выполнялось равенство

$$
\mathbf{P}\left\{\varepsilon_{k, t}=\varepsilon_{0}, \varepsilon_{k, t+h}=\varepsilon_{1}, \ldots, \varepsilon_{k, t+(x-1) h}=\varepsilon_{x-1}\right\}=r^{\varepsilon_{x}^{+}} l^{\varepsilon_{x}^{-}},
$$

где $r^{\varepsilon_{x}^{+}} l^{\varepsilon_{x}^{-}}=r^{\varepsilon_{0}+\varepsilon_{1}+\cdots+\varepsilon_{x-1}} l^{e-\left(\varepsilon_{0}+\varepsilon_{1}+\cdots+\varepsilon_{x-1}\right)}$, необходимо и достаточно, чтобы $і$-я частица была абсолютным лидером.

Д ок аз а тель с тв о. Н е о бходи м о с ь следует из леммы 5.1.

Д о с т а т о ч н о с т ь. Допустим, что $s$-я частица является лидером. Докажем, что $k$-я $(k \neq s)$ частица блуждает с параметрами $r, l$, т.е. выполнено (5.2).

Введем обозначение

$$
\begin{gathered}
A_{m, t}=\left\{\rho_{k, t}+\rho_{k+1, t}+\cdots+\rho_{s-1, t}=m\right\}=\left\{\mu_{t}(k, s)=m\right\} \\
m=s-k, \ldots, n-s+k .
\end{gathered}
$$


Так как $\mathbf{P}\left\{A_{m+1, t}, A_{m, t+1}\right\}=\mathbf{P}\left\{A_{m, t}, A_{m+1, t+1}\right\}, m=s-k, \ldots, n-s+k$, то

$$
\mathbf{P}\left\{A_{m+1, t}, \varepsilon_{k, t}=d, \varepsilon_{s, t}=0\right\}=\mathbf{P}\left\{A_{m, t}, \quad \varepsilon_{k, t}=0, \varepsilon_{s, t}=d\right\}
$$

для $m=s-k, \ldots, n-s+k-1$. Методом математической индукции покажем, что

$$
\begin{aligned}
\mathbf{P}\left\{\varepsilon_{k, t}=\varepsilon_{0}, \varepsilon_{k, t+h}\right. & \left.=\varepsilon_{1}, \ldots, \varepsilon_{k, t+(x-1) h}=\varepsilon_{x-1}, A_{m, t+x h}\right\} \\
& =r^{\varepsilon_{x}^{+}} l^{\varepsilon_{x}^{-}} \mathbf{P}\left\{A_{m, t+x h}\right\} .
\end{aligned}
$$

Для $x=1$ имеем:

$$
\begin{aligned}
\mathbf{P}\left\{\varepsilon_{k, t}=1, A_{m, t+h}\right\}=\mathbf{P}\left\{\varepsilon_{k, t}=1, \varepsilon_{s, t}=1, A_{m, t}\right\} \\
\quad+\mathbf{P}\left\{\varepsilon_{k, t}=1, \varepsilon_{s, t}=0, A_{m+1, t}\right\}=\mathbf{P}\left\{\varepsilon_{k, t}=1, \varepsilon_{s, t}=1, A_{m, t}\right\} \\
\quad+\mathbf{P}\left\{\varepsilon_{k, t}=0, \varepsilon_{s, t}=1, A_{m, t}\right\}=\mathbf{P}\left\{\varepsilon_{s, t}=1, A_{m, t}\right\} \\
=\mathbf{P}\left\{\varepsilon_{s, t}=1\right\} \mathbf{P}\left\{A_{m, t}\right\}=r \mathbf{P}\left\{A_{m, t}\right\} .
\end{aligned}
$$

Допустим, что утверждение верно для $x$. Докажем его для $x+1$ :

$$
\begin{aligned}
\mathbf{P}\{ & \left.\varepsilon_{k, t}=\varepsilon_{0}, \varepsilon_{k, t+h}=\varepsilon_{1}, \ldots, \varepsilon_{k, t+x h}=1, A_{m, t+(x+1) h}\right\} \\
= & \mathbf{P}\left\{\varepsilon_{k, t}=\varepsilon_{0}, \ldots, \varepsilon_{k, t+x h}=1, \varepsilon_{s, t+x h}=1, A_{m, t+x h}\right\} \\
& +\mathbf{P}\left\{\varepsilon_{k, t}=\varepsilon_{0}, \ldots, \varepsilon_{k, t+x h}=1, \varepsilon_{s, t+x h}=0, A_{m+1, t+x h}\right\} \\
= & r^{\varepsilon_{x}^{+}} l^{\varepsilon_{x}^{-}}\left[\mathbf{P}\left\{\varepsilon_{k, t+x h}=1, \varepsilon_{s, t+x h}=1, A_{m, t+x h}\right\}\right. \\
& \left.\quad+\mathbf{P}\left\{\varepsilon_{k, t+x h}=1, \varepsilon_{s, t+x h}=0, A_{m+1, t+x h}\right\}\right] \\
=r^{\varepsilon_{x}^{+}} l^{\varepsilon_{x}^{-}} & {\left[\mathbf{P}\left\{\varepsilon_{k, t+x h}=1, \varepsilon_{s, t+x h}=1, A_{m, t+x h}\right\}\right.} \\
& \left.\quad+\mathbf{P}\left\{\varepsilon_{k, t+x h}=0, \varepsilon_{s, t+x h}=1, A_{m, t+x h}\right\}\right] \\
= & r^{\varepsilon_{x}^{+}} l^{\varepsilon_{x}^{-}} \mathbf{P}\left\{\varepsilon_{s, t+x h}=1, A_{m, t+x h}\right\}=r^{\varepsilon_{x}^{+}} l^{\varepsilon_{x}^{-}} \mathbf{P}\left\{\varepsilon_{s, t+x h}=1\right\} \mathbf{P}\left\{A_{m, t+x h}\right\} \\
= & r^{\varepsilon_{x}^{+}} l^{\varepsilon_{x}^{-}} r \mathbf{P}\left\{A_{m, t+x h}\right\} .
\end{aligned}
$$

Итак, получаем, что для любых $n$ и $m(s-k \leqslant m \leqslant n-s+k)$

$$
\mathbf{P}\left\{\varepsilon_{k, t}=\varepsilon_{0}, \varepsilon_{k, t+h}=\varepsilon_{1}, \ldots, \varepsilon_{k, t+x h}=1, A_{m, t+(x+1) h}\right\}=r^{\varepsilon_{x}^{+}} l^{\varepsilon_{x}^{-}} r \mathbf{P}\left\{A_{m, t+x h}\right\} .
$$

Суммируя по $m(s-k \leqslant m \leqslant n-s+k)$, приходим к (5.2). Теорема 5.1 доказана.

3 а м е ч а н и е 5.2. Результат теоремы 5.1 можно трактовать следующим образом. Если одну частицу сделать видимой, а остальные невидимыми, то видимая частица совершает случайное блуждание с теми же параметрами, что и частица-лидер. 
Следствие 5.1. Если $s=2$, то из существования абсолютного лидера следует равенство $r_{n-1}=1$ и описанная модель совпадает $c$ моделью 2.1. Таким образом, из теоремь 5.1 следуют результаты, описаннье в разделе 2.

Рассмотрим следующие утверждения:

(a) $s$-я частица является лидером первого типа;

(b) $s$-я частица является лидером второго типа;

(c) каждая отдельно рассматриваемая частица совершает случайное биномиальное блуждание с параметрами $r, l$.

Следствие 5.2. При выполнении условий леммь 5.1 верны следующие импликации:

$$
(\mathrm{a}) \&(\mathrm{~b}) \Rightarrow(\mathrm{c}), \quad(\mathrm{a}) \&(\mathrm{c}) \Rightarrow(\mathrm{b}), \quad(\mathrm{b}) \&(\mathrm{c}) \Rightarrow(\mathrm{a})
$$

$(\mathrm{a}) \&(\mathrm{~b}) \Leftrightarrow(\mathrm{a}) \&(\mathrm{c}), \quad(\mathrm{a}) \&(\mathrm{~b}) \Leftrightarrow(\mathrm{b}) \&(\mathrm{c}), \quad(\mathrm{a}) \&(\mathrm{c}) \Leftrightarrow(\mathrm{b}) \&(\mathrm{c})$.

6. Примеры. Пусть число $n$ точек на окружности равно 40 и число частиц $s$ принимает значения от 2 до 40.

М о д е ль 6.1. Модель с одним абсолютным лидером. Время движения $T=100000 h$. Показатель эффективности - пропускная способность системы $(\lambda)$, т.е. число частиц, проходящих через фиксированную точку в единицу времени. Так как имеется абсолютный лидер, то средняя скорость движения системы всегда равна $r$. Следовательно, с ростом числа частиц линейно растет и пропускная способность.

М о д е ль 6.2. Симметричная модель. Пусть

$$
r_{k}^{i}=0.7, \quad l_{k}^{i}=1-r_{k}^{i}=0.3, \quad i=1,2, \ldots, s, \quad k=1, \ldots, n-s+1 .
$$

На рисунке приводится диаграмма для такой модели.

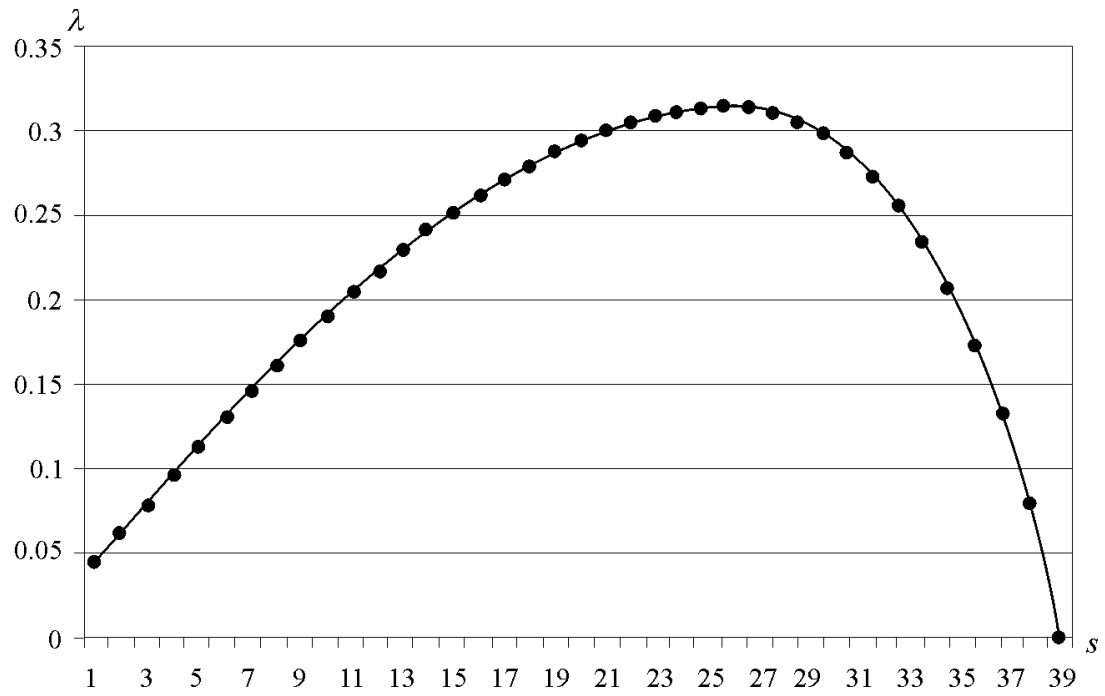

Рис. Пропускная способность для симметричной модели 
Для расчета пропускной способности $(\lambda)$ используется результат о случайном блуждании отдельно рассматриваемой частицы.

С увеличением плотности движения (числа движущихся частиц) до некоторого уровня пропускная способность дороги растет. Начиная с некоторого значения, с ростом плотности движения пропускная способность системы падает, и возникает явление «транспортной пробки». Поэтому, используя предложенный подход в зависимости от параметров можно оценить значение плотности движения, когда пропускная способность принимает максимальное значение, а также оценить значение плотности движения, когда возникает «транспортная пробка». Поэтому во многих странах на высокоскоростных дорогах временно сужают дорогу (уменьшая тем самым плотность движения), чтобы далее избежать возникновения «транспортной пробки».

\section{СПИСОК ЛИТЕРАТУРЫ}

1. Беляев Ю. К. Об упрощенной модели движения без обгона. - Изв. АН СССР., Техн. киберн., 1969, № 3, с. 17-21.

2. Целе У. Обобщение модели движения без обгона. - Изв. АН СССР, Техн. киберн., 1972, № 5, с. 100-103.

3. Гаджиев А. Г. Стохастические челночные системы. - Докл. РАН, 2001, т. 380, № 5 , с. $583-584$.

4. Секей Г. Парадоксы в теории вероятностей и математической статистике. М.: Мир, 1990, 240 с.

5. Crane M.A. Queues in transportation systems II: An independently dispatched system. - J. Appl. Probab., 1974, v. 2, № 1, p. 145-158.

6. Ignall E., Kolesar P. Optimal dispatching of an infinite-capacity shuttle: Control at a single terminal. - Oper. Res., 1974, v. 22, p. 1008-1024.

7. Newell G.F. Control of pairing of vehicles on a public transportation route, two vehicles, one control point. - Transportation Sci., 1974, v. 8, № 4, p. 248-264.

8. Gadżiev A.G. (Hajiyev A.H.) Delays reducing the waiting time in queueing systems with cyclic services. - Scand. J. Statist., 1985, v. 12, p. 301-307.

9. Беляев Ю.К., Буслаев А.П., Селезнев О.В. и др. Марковская аппроксимация стохастической модели движения по двум полосам. Отчет № 1234-В2002. М.: Московский автомобильно-дорожный институт, 2002,32 с.

10. Гаджиев А.Г. Модель движения частиц без обгона по замкнутому контуру. Изв. АН СССР, Техн. киберн., 1976, № 5, с. 79-84.

11. Гаджиев А.Г. О случайном блуждании частиц по кольцу. - Матем. заметки, 1990, т. 47, № 6 , с. 140-143.

12. Ширяев А.Н. Вероятность-1. М: МЦНМО, 2004, 519 с.

13. Гаджиев А. Г., Джафарова Х. А., Маммадов Т. Ш. Математические модели движущихся частиц без обгона. - Докл. РАН, 2010, т. 432, № 3, с. 304-307.

Поступила в редакцию 2.VIII.2011 